Arq. Bras. Med. Vet. Zootec., v.59, n.2, p.513-516, 2007

\title{
Comunicação
}

[Communication]

\section{Uretrostomia para colheita seriada de urina em bezerros}

\author{
[Urethrostomy for serial sampling of urine in calves] \\ R.V. Flôres ${ }^{1}$, C.A. Souza ${ }^{2}$, V.A. Gheller ${ }^{3}$, V. Foltynek ${ }^{1}$, C.A. Marval ${ }^{1}$, A.I. Bordin ${ }^{1}$ \\ A.A.F. Viana ${ }^{1}$, N.M. Ocarino ${ }^{3}$, R. Serakides ${ }^{3} *$ \\ ${ }^{1}$ Aluno de pós-graduação - EV-UFMG - Belo Horizonte, MG \\ ${ }^{2}$ Aluna de graduação - EV-UFMG - Belo Horizonte, MG \\ ${ }^{3}$ Escola de Veterinária - UFMG \\ Caixa Postal 567 \\ 30123-970 - Belo Horizonte, MG.
}

Estudos da função renal em bovinos têm sido realizados para a determinação do perfil metabólico e para a detecção precoce de doenças metabólicas, utilizando-se cateterismo vesical (Fleming et al., 1991). Em bezerros, a utilização de gaiolas metabólicas permite obter urina para a determinação do ritmo de filtração glomerular e do controle da natremia (Skrzypczak e Drzezdson, 2001). Mas a necessidade de se obter o volume urinário preciso e as concentrações de solutos após infusão parenteral de soluções eletrolíticas nos tratamentos de desidratação requer a utilização do cateterismo vesical, que, por vias anatômicas, em machos bovinos, é de difícil consecução. O objetivo deste estudo é propor uma técnica de implantação cirúrgica de cateter vesical em bezerros.

Foram utilizados 27 bezerros da raça Holandesa, machos, com idades entre oito e 16 dias e peso médio de $39,50 \pm 4,80 \mathrm{~kg}$. A temperatura ambiente mínima mensurada com termômetro de bulbo seco foi de $20^{\circ} \mathrm{C}$ e a máxima de $27^{\circ} \mathrm{C}$, e a umidade relativa do ar esteve entre $58 \%$ e $62 \%$. Os animais foram alojados em gaiolas individuais com $1,12 \mathrm{~m}$ de largura, $1,50 \mathrm{~m}$ de comprimento e $1,12 \mathrm{~m}$ de altura, suspensas $20 \mathrm{~cm}$ do solo e com piso de madeira ripado. A cama consistiu de feno de tifton de baixa qualidade, limpa diariamente e trocada a cada dois dias. A água foi fornecida ad libitum em baldes plásticos de oito litros, mensurada em

Recebido em 22 de novembro de 2005 Aceito em 22 de dezembro de 2006

*Autor para correspondência (corresponding author)

E-mail: serakide@dedalus.lcc.ufmg.br provetas de $1000 \mathrm{ml}^{1}$ e trocada duas vezes/dia. A alimentação consistiu de raspa de leite ${ }^{2}$ diluída em água entre $36^{\circ} \mathrm{C}$ e $40^{\circ} \mathrm{C}(130 \mathrm{~g} / \mathrm{l})$, acrescida de $250 \mathrm{ml}$ de colostro por refeição por animal/dia, totalizando o volume diário de quatro litros/animal/dia e fornecida nas mamadeiras em duas refeições diárias às 8 e 16h. Os animais foram adquiridos com idades entre um e nove dias e passaram por período de sete dias de adaptação às instalações, à dieta e às condições ambientais. $\mathrm{O}$ experimento foi aprovado pelo Comitê de Ética em Experimentação Animal da UFMG.

Durante todo o período de adaptação, os animais foram submetidos ao exame físico completo, seguindo técnica descrita por Dirksen et al. (1993) a cada $12 \mathrm{~h}$. Os exames pré-cirúrgicos consistiram no exame físico completo, determinação do peso corpóreo, hemograma completo, proteínas séricas totais, albumina, creatinina e fósforo séricos e urinálise, todos realizados 48 horas antes da cirurgia.

As sondas urinárias ${ }^{3}$ de polivinil atóxico (PVC) siliconizado com conector e tampa, furos laterais e ponta fechada foram confeccionadas especialmente para este experimento.

As sondas foram implantadas segundo a técnica de uretrostomia (Larson, 1996). Os animais foram contidos em decúbito dorsal com os membros posteriores em flexão após a aplicação

\footnotetext{
${ }^{1}$ Proveta plástica $(1000 \mathrm{ml})$ J.Prolab Ind.Com.Prod. para Laboratório Ltda, São José dos Pinhais, PR, Brasil

${ }^{2}$ Leite integral - Itambé, Sete Lagoas, MG, Brasil

${ }^{3}$ Sonda uretral descartável - Fabrimed, Santo Antonio de Posse, MG, Brasil
} 
intramuscular de cloridrato de xilazina a $2 \%{ }^{4}$ na dose de $0,15 \mathrm{mg} / \mathrm{kg}$ de peso corporal. A tricotomia da região perineal foi realizada desde o ânus até o escroto, seguindo-se antissepsia e anestesia local com cloridrato de lidocaína a $2 \%{ }^{5}$. Uma incisão de aproximadamente $3 \mathrm{~cm}$ foi feita na pele, acima do arco isquiádico, próxima ao ânus e na fáscia, possibilitando a exposição dos músculos retratores do pênis. Esses músculos foram dissecados para a exposição do músculo bulboesponjoso e a localização da uretra para a uretrostomia e inserção de aproximadamente $40 \mathrm{~cm}$ da sonda até a bexiga urinária (Fig. 1A-C). A porção da uretra peniana caudal à incisão foi obstruída com sutura. A fixação da sonda no prepúcio foi realizada através de sutura e fio de náilon $2-0^{6}$; sua extremidade livre foi fixada na face caudal do membro posterior esquerdo com pontos simples separados e fio de náilon (Fig. 1D). O conector foi acoplado ao coletor urinário em sistema fechado $(2000 \mathrm{ml})$ drenável $^{7}$ (Fig. 2A e B). A ferida cirúrgica foi protegida por esparadrapo apropriado ${ }^{8}$.

O pós-operatório consistiu na avaliação clínica dos animais a cada $12 \mathrm{~h}$ e realização de hemograma completo, determinação da creatinina e fósforo séricos, proteínas totais, albumina e urinálise, $36 \mathrm{~h}$ após a uretrostomia.

O período de $36 \mathrm{~h}$ foi aguardado após a cirurgia para o início da colheita de urina. A bexiga foi esvaziada e feita conexão do coletor urinário à extremidade da sonda. Os animais foram avaliados durante quatro dias após a cirurgia.

A avaliação consistiu também no monitoramento contínuo do fluxo urinário através da sonda a fim de se evitar interrupções por eventual estrangulamento de sua extremidade livre, no esvaziamento do coletor urinário antes de atingir sua capacidade máxima $(2000 \mathrm{ml})$, na manutenção do esparadrapo recobrindo a ferida cirúrgica durante o período mínimo de 48 horas e na mensuração do volume de urina produzido após o esvaziamento vesical completo no tempo $=0 \mathrm{~h}$ e obtenção de alíquotas em intervalos de tempos préfixados: períodos $(\mathrm{P}=0-0,5 \mathrm{~h} ; \mathrm{P}=0,5-1,0 \mathrm{~h} ; \mathrm{P}=1,0$ -

\footnotetext{
${ }^{4}$ Anasedan injetável - Agribrands do Brasil Ltda, Paulínia, SP, Brasil

${ }^{5}$ Cloridrato de lidocaína $2 \%$ sem vaso - Hypofarma, Ribeirão das Neves, MG, Brasil

${ }^{6}$ Fio de sutura náilon monofilamento 2-0 - Technofio AceIndústria Comércio Ltda, Goiânia, GO, Brasil

${ }^{7}$ Coletor de urina sistema fechado transparente $(2000 \mathrm{ml})$ drenável - Collector Ind.Com.de Prod.Hospitalares Ltda, Bauru, SP, Brasil

${ }^{8}$ Transpore - 3M do Brasil Ltda, Sumaré, SP, Brasil
}

2,0h; $\mathrm{P}=2,0-8,0 \mathrm{~h} ; \mathrm{P}=8,0-24,0 \mathrm{~h})$ drenando-se o coletor urinário ou através de punção da urina diretamente da bexiga urinária, acoplando-se a extremidade da seringa de $20 \mathrm{ml}$ na extremidade da sonda. Sempre que necessária, foi realizada injeção de ar no interior da bexiga para provocar contração e auxiliar na drenagem de pequenos volumes (Fleming et al., 1991).

Os animais foram submetidos à eutanásia ao final do período experimental, pela aplicação de uma superdosagem de pentobarbital $(60 \mathrm{mg} / \mathrm{kg}$, via EV). À necropsia foi avaliada a presença de retenção urinária e de alterações decorrentes de retenção de urina tais como hidronefrose, pielonefrites etc e se a sonda urinária permanecia intacta no interior da bexiga.

Foi utilizado o teste $t$ de Student pareado para a comparação dos dados obtidos antes (48h) e depois (36h) da realização da uretrostomia.

As sondas urinárias, com $80 \mathrm{~cm}$ de comprimento, foram confeccionadas visando obter maior rigidez e menor flexibilidade. Em 19 dos bezerros utilizados neste estudo, utilizou-se a sonda urinária $\mathrm{n}^{\circ} 10$, em cinco bezerros a de $n^{\circ} 08$ e em três bezerros a de $n^{\circ}$ 06.

Baseando-se no exame físico completo, na determinação do peso corporal, no hemograma completo, na determinação da creatinina e fósforo sérico, proteínas totais, albumina e na urinálise realizados 48 horas antes e 36 horas após a cirurgia, pode-se afirmar que o pós-operatório evoluiu sem qualquer complicação clínica em 26 dos 27 animais estudados. Um dos animais apresentou diarréia e foi retirado do experimento. Outro animal apresentou hematúria nas primeiras 12 horas do pós-operatório e, após 24 horas, não houve nenhuma alteração à urinálise. Para nenhuma variável houve qualquer diferença quando os tempos foram comparados. Não houve diferença significativa no hemograma ou nas variáveis bioquímicas do sangue. Em nenhum momento as médias diferiram dos valores considerados normais para bezerros (Kaneko, 1989; Dirksen et al., 1993; Goff, 2000).

Dos 27 animais utilizados neste estudo, 22 apresentaram resultados satisfatórios de acordo com os critérios avaliados. Cinco bezerros apresentaram: hidronefrose unilateral moderada à necropsia (dois animais), obstrução do fluxo urinário por estrangulamento da sonda urinária em sua fixação externa cutânea, observada durante o período de colheita de urina (dois animais) e diarréia profusa após a cirurgia (um animal). 

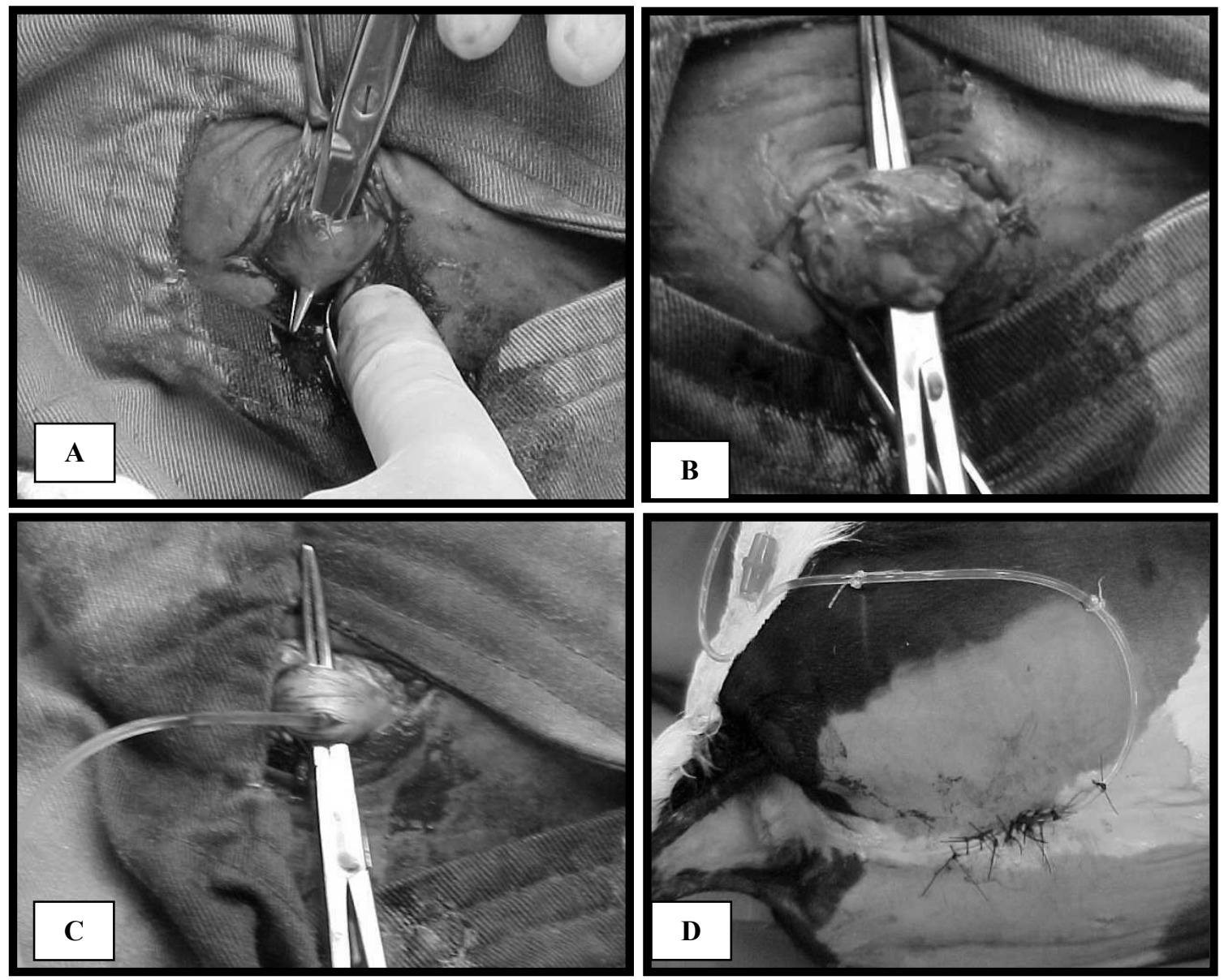

Figura 1. Bezerro, uretrostomia. A) Incisão de aproximadamente $3 \mathrm{~cm}$ na pele, acima do arco isquiádico, próxima ao ânus e na fáscia, com exposição dos músculos retratores do pênis. B) Dissecação e exposição do músculo bulboesponjoso. C) Uretrostomia e inserção de aproximadamente $40 \mathrm{~cm}$ da sonda até a bexiga urinária. D) Fixação da extremidade externa da sonda uretral na face caudal do membro posterior.

Aconselha-se que a extremidade externa da sonda uretral seja fixada retilineamente na face caudal do membro posterior, com o cuidado de se evitar a formação de alças que possam provocar seu estrangulamento e comprometer o fluxo urinário contínuo, mantendo-se sua extremidade de conexão ao coletor urinário até a altura da articulação tibiotársica, evitando-se que enrole no membro do animal. Devido a sua menor flexibilidade quando comparada às sondas uretrais comerciais, não houve dificuldades em sua passagem no arco isquiádico durante a inserção até $\mathrm{o}$ interior da bexiga, tendo cada cirurgia duração média de 40 minutos.

Utilizando-se a técnica descrita, o fluxo urinário é contínuo, necessitando-se, portanto, de coletores urinários a fim de evitar o umedecimento das partes inferiores dos membros posteriores dos animais e causar seu desconforto. $\mathrm{O}$ volume de urina produzido por animal é variável, principalmente em função do consumo de água. O volume de água consumido no período de tempo igual a 20 horas foi de $1752,92 \mathrm{ml} \pm 2333,22$. O volume urinário médio por animal no período de 12 horas de coleta urinária foi igual a $1797,1 \pm 1115,7 \mathrm{ml}$, ressaltandose a necessidade de drenagem freqüente do coletor urinário com capacidade para $2000 \mathrm{ml}$. Os coletores urinários foram mantidos na cama dos animais, sem qualquer inconveniente ou inaceitação dos mesmos (Fig. 2).

Conclui-se que uretrostomia permite a colheita urinária em curtos intervalos, com drenagem completa da urina produzida e contida na bexiga urinária, proporcionando a mensuração do volume de urina produzido e a obtenção de alíquotas em tempos pré-fixados.

Palavras-chave: bezerro, cateterismo, urestrotomia 

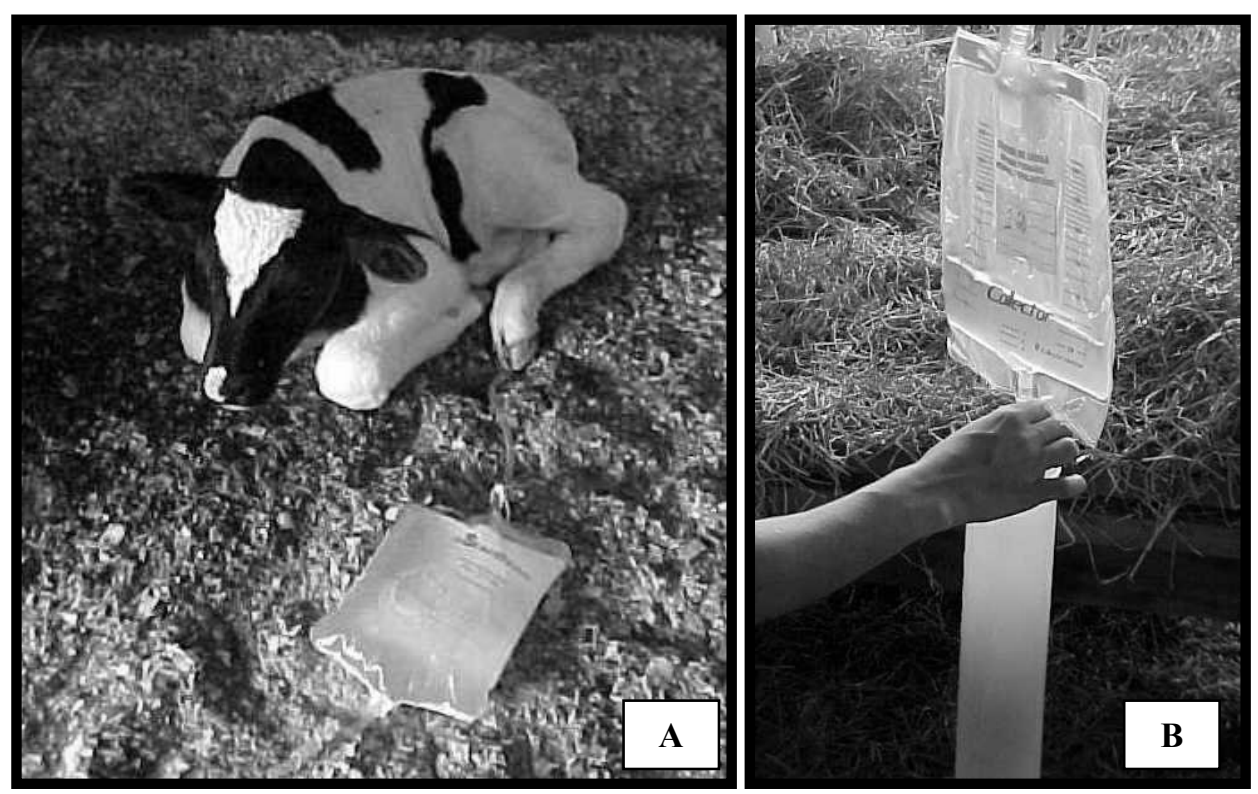

Figura 2. A) Bezerro com conector da sonda urinária acoplada ao coletor em sistema fechado (2000ml) drenável. B) Esvaziamento do coletor de urina.

\section{ABSTRACT}

Urethrostomy for serial sampling of urine was used due to the necessity of one technique that allowed the total urinary collection in definite time periods. Twenty-seven male Holstein calves from 8 to 16-days-age having a mean weight $39.50 \pm 4.80 \mathrm{~kg}$ were used. Urethrostomy was carried out with the use of urethral sounding lead that was made for this purpose. In agreement with the clinical examinations, including seric biochemical, urinalysis and blood test and postmortem examination, the technique achieved $81.5 \%$ of satisfactory results.

Keywords: calf, catheterism, urethrostomy

\section{REFERÊNCIAS BIBLIOGRÁFICAS}

DIRKSEN, G.; GRUNDER, H.D.; STOBER, M. Exame clínico dos bovinos. 3.ed. Rio de Janeiro: Guanabara Koogan, 1993. 419p.

FLEMING, S.A.; HUNT, E.L.; RIVIERE, J.E. et al. Renal clearance and fractional excretion of electrolytes over four 6-hour periods in cattle. Am. J. Vet. Res., v.52, p.5-8, 1991.

GOFF, J.P. Pathophysiology of calcium and phosphorus disorders. Vet. Clin. N. Am.: Food Anim. Pract., v.16, p.319-337, 2000.

KANEKO, J.J. (Ed.). Clinical biochemistry of domestic animals. 4.ed. San Diego: Academic, 1989. 900p.

LARSON, B.L. Identifying, treating, and preventing bovine urolithiasis. Vet. Med.: Food Anim.Pract., v.12, p.366-377, 1996.

LUNN, P.D.; McGUIRK, S.M. Renal regulation of electrolyte and acid-base balance in ruminants. Vet. Clin. N. Am.: Food Anim. Pract., v.1, p.1-27, 1985.
MICHELL, A.R. Understanding fluid therapy. Ir. Vet. J., v.37, p.94-103, 1983.

NEIGER, R.D.; HAGEMOSER, W.A. Renal percent clearance ratios in cattle. Vet. Clin. Pathol., v.14, p.31-35, 1985.

SKRZYPCZAK, W.F.; DRZEZDZON, D. Kidneys adaptation of kid and calf newborns to natremia regulation. Electr. J. Pol. Agric. Univ., v.4, p. _, 2001. Disponível em:

http://www.ejpau.media.pl/series/volume4/issue2/veterina ry/art-02.html > . Acessado em: 23/08/2003.

TYLER, J.W.; WELLES, E.G.; ERSKINE, R.J. et al. Clinical and clinicopathologic changes in cows with endotoxin-induced mastitis treated with small volumes of isotonic or hypertonic sodium chloride administered intravenously. Am. J. Vet. Res., v.55, p.278-287, 1994. 